\title{
Algunos interrogantes sobre integrar las prácticas académicas: Reflexiones y Propuestas
}

\section{Some questions about integrating academic practices: Reflections and Proposals}

\author{
Carlos A. Zavaro \\ https://orcid.org/0000-0003-3298-7383 \\ czavaro@fcnym.unlp.edu.ar \\ Facultad de Ciencias Naturales y Museo | UNLP | Argentina \\ Facultad de Ciencias Exactas y Naturales I UB | Argentina
}

\section{RESUMEN}

Las prácticas integrales constituyen un formato que articula funciones académicas como la docencia, la investigación y la extensión bajo una síntesis que las excede y que, a su vez, integra disciplinas y actores sociales de la comunidad con las demandas en torno a los problemas que atraviesan el territorio y que constituyen insumos de la práctica. Algunas de las reflexiones y propuestas que conforman estas líneas recuperan aportes de una diversidad de experiencias que contribuyen a delinear colectivamente las trayectorias de un espacio que en sí mismo se vislumbra como académicamente revolucionario, por promover un aprendizaje situado con el propósito de formar sujetos integrales y comprometidos con la realidad y con la comunidad en la que aprenden y viven.

\section{ABSTRACT}

Integral practices constitute a format that combines academic functions such as teaching, research and extension under a synthesis that goes beyond its. Many disciplines and the actors of the community with the problems that cross these territory are also incorporated as insume of the practice. Some of the reflections and proposals that make up these lines, recover the contributions of a diversity of experiences that have been contributed to delineate the trajectories of a space that in itself is seen as academically revolutionary, by promoting situated learning whit the purpose to form integral subjects committed to reality and the community in which they learn and live.

PALABRAS CLAVE

territorio, investigación, extensión, docencia

\section{KEY WORDS}

territory,

research,

extension, teaching. 


\section{PRESENTACIÓN}

Las prácticas integrales han ido ganando en los últimos años la centralidad del debate en un sector de la universidad argentina que, consciente de las tradiciones que han caracterizado a la gestión disociada del conocimiento, ha comenzado a impulsar una corriente instituyente (Kaplún, 2014) en la que la investigación, la extensión y la docencia son interpeladas incentivando un formato en el que éstas se intersectan, aun sin que esas intersecciones desconozcan las trayectorias que las definen. Este propósito de integrar las prácticas parecería una utopía en una institución hostil a las innovaciones (Brunner y Flisfisch, 1989) y en la que existe una diversidad de intereses que, muchas veces, tensionan la toma de decisiones en los espacios de cogobierno.

\section{la investigación, la extensión y la docencia son interpeladas incentivando un formato en el que éstas se intersectan, aun sin que esas intersecciones desconozcan las trayectorias que las definen.}

Si bien el tema ocupa cierta centralidad en la agenda universitaria, y existen diversas experiencias que comienzan a configurar el formato en el que esa integralidad encuentra forma y sentido, los espacios en que se desarrollan parecieran aún ser aislados y estar sujetos a los aportes de quienes lo integran, planifican y llevan a término, lo que no sólo contribuye a su heterogeneidad, sino que constituye una oportunidad para el intercambio de experiencias y el debate en el que éstas van definiéndose y configurando. Reflexionar sobre este tipo de prácticas, que comienzan a vislumbrar un escenario de cambio curricular, en mi opinión, profundamente revolucionario, constituye el propósito 
de estas líneas sobre la base de algunas preguntas que van sucediéndose como forma de articular las ideas.

\section{¿Qué entendemos por prácticas integrales?}

Las prácticas integrales constituyen un espacio en construcción. Un formato en el que es posible integrar prácticas instituidas como funciones académicas entre las que la investigación, la extensión y la docencia, tradicionalmente han sido concebidas de manera disociada por las tradiciones, los propósitos y las metodologías que las delimitan, pero sobretodo por las estructuras organizacionales en las que ancoran, aunque tal como afirman Tommasino y Rodríguez (2010) la integralidad no se reduce únicamente a esa articulación.

Las prácticas integrales constituyen un espacio en construcción. Un formato en el que es posible integrar prácticas instituidas como funciones académicas entre las que la investigación, la extensión y la docencia, tradicionalmente han sido concebidas de manera disociada por las tradiciones, los propósitos y las metodologías que las delimitan, pero sobretodo por las estructuras organizacionales en las que ancoran

\section{¿Qué las distingue?}

Bajo la concepción disociativa, la investigación suele centrarse en preguntas a las que se pretende responder a partir de la formulación de hipótesis que son (o no) corroboradas utilizando diferentes metodologías (Chalmers, 2000), mientras que sus resultados son puestos a consideración de una comunidad que, en base a una cultura particular de relacionarse con el saber, y con las lógicas con las que se construye, las legitima (Sandoval et al, 2004) a partir de su aceptación y publicación en revistas especializadas evaluadas con indicadores cienciométricos de impacto (Millán et al, 2017).

La extensión, por otra parte, ha fortalecido el vínculo con la comunidad a través de propuestas que, si bien están diseñadas bajo la premisa de dar respuesta a las demandas territoriales (López, 2011), no siempre incorporan la perspectiva comunitaria y transcurren por andariveles paralelos a la investigación, aunque en algunos casos puedan anclar en una misma problemática.

La docencia por su parte, pareciera haberse convertido en contexto de aplicación del conocimiento producido por la ciencia a partir de una transferencia que es entendida como transposición de la didáctica (Chevallard, 1999) y en la que el docente universitario opera como 
curador del saber, eligiendo los temas que considera relevantes para la formación de los futuros egresados y diseñando mediaciones de ese aprendizaje y modos de decodificar el conocimiento encriptado por el lenguaje disciplinar.

Este modo de definir las prácticas y de caracterizarlas como compartimentos estancos, sucumbe ante la concepción de la integralidad, que sin ignorar la existencia de tradiciones y hábitos (Bourdieu, 1984) que han ido delineando los modos de entender el trabajo académico, apuestan a integrar funciones y metodologías, no como sumatoria de las anteriores, sino como una síntesis que integra a actores, experiencias y modos de objetivación, que encuentran escenario y contexto en la experiencia pedagógica.

\section{¿Por qué un formato pedagógico como escenario?}

La enseñanza es el escenario donde concebir un formato en que puedan integrarse las funciones académicas porque la articulación entre éstas tiene una connotación profundamente pedagógica, en tanto constituye una experiencia que se crea y recrea en el contexto educativo (Zavaro, 2019). Si la docencia elude la transmisión acrítica del saber para focalizarse en la formación de las generaciones futuras en un campo de conocimiento determinado, pero entendido como un espacio de intercambio entre sujetos atravesado por saberes conceptuales que encierran historia, epistemología, método y lenguaje, entonces, integrar las funciones implicaría más que transmitir un saber instituido, resignificar la historia de esos procesos que han llevado a consolidar los aportes conceptuales que hoy son legitimados, pero entendiéndolos desde la lógica y las metodologías que los han producido, aunque transformados en herramienta didáctica para su comprensión.

\section{La enseñanza es el escenario donde concebir un formato en que puedan integrarse las funciones académicas porque la articulación entre éstas tiene una connotación profundamen- te pedagógica, en tanto constituye una experiencia que se crea y recrea en el contexto educativo}

Las prácticas integrales entonces, deberían promover la integración de las prácticas disociadas de tal manera que, en principio, la docencia pueda estar atravesada por la lógica que opera en la producción de conocimientos, reformulando la educación transferencista. Esta posición, claramente constructivista (Porlán, 2002), no sólo contribuye a reconvertir la práctica docente en un formato pensado desde el hacer, sino en un hacer pensado en contexto. Es el territorio, y la comunidad que lo habita, quien garantiza la contextualización y fomenta, con im- 
pronta extensionista, la integración entre los actores que conforman el espacio, promoviendo la circulación de un saber que horada prefiguraciones e imaginarios y visibiliza tanto, las experiencias personales, como el conocimiento adquirido y adherido a las trayectorias académicas y a las vivencias personales.

\section{Es el territorio, y la comunidad que lo habita, quien garantiza la contextualización y fomenta, con impronta extensionista, la integración entre los actores que conforman el espacio, promo- viendo la circulación de un saber que horada prefiguraciones e imaginarios y visibiliza tanto, las experiencias personales, como el conocimiento adquirido y adherido a las trayectorias académicas y a las vivencias personales.}

\section{¿Resistencias o prejuicios?}

A pesar de las ventajas, estos espacios aún son resistidos en la academia. Algunas de las resistencias pueden asociarse a prejuicios infundados en el temor de que los contenidos no sean abordados en profundidad. Quizás en algunas disciplinas y en particular en aquellas que ocupan la grilla de las materias que integran los ciclos básicos, suele rechazarse todo aquello que implique romper con la tradición de enseñar contenidos conceptuales que tienen correlato únicamente en trabajos prácticos, en experiencias de laboratorio o en seminarios y debates. Esas decisiones, quizás, tampoco sean desacertadas en cuanto ese cuerpo conceptual pueda constituir el pilar en que se sostengan -en otros espacios- otras formas de diálogo y de apropiación del conocimiento.

Otro prejuicio remite a la importancia de que sea en el docente y no otro, el responsable de explicar contenidos, aunque un aprendizaje significativo no siempre está mediado por un profesor que explique como si los estudiantes no pudiesen aprender por sí mismos. Existen diversos modelos que rompen con esa concepción de la enseñanza en la que la explicación se convierte en un modo de obturar la razón y la capacidad de reflexionar, de interpelar e interpelarse, preguntar y preguntarse, e incluso de dudar de lo enseñado, aun cuando no se dude de quien lo enseña.

\section{¿Cuál es el rol de docentes y estudiantes ante esta encrucijada?}

Algunos modelos que han comenzado a emerger en medio de la pandemia ocasionada por el coronavirus y de la reconversión, casi forzosa, de la enseñanza tradicional en una educación de tipo invertida (Andrade y Chacón, 2018) que permitió explorar nuevas posibilidades, dan cuenta de la relevancia que tienen los formatos donde el protago- 
nismo recae en la figura del estudiante y en su capacidad de explorar alternativas de relación con el saber, más que en la centralidad del docente "explicador"que hegemoniza la palabra. Un docente reconvertido en facilitador, que aclara dudas, fomenta debates, promueve la circulación de la palabra y otorga los permisos para escudriñar en aquellos contenidos que eran presentados como indiscutibles, es central en estos nuevos formatos y constituye un garante de las prácticas integrales.

\section{Un docente reconvertido en facilitador, que aclara dudas, fo- menta debates, promueve la circulación de la palabra y otorga los permisos para escudriñar en aquellos contenidos que eran presentados como indiscutibles, es central en estos nuevos for- matos y constituye un garante de las prácticas integrales.}

Paradójicamente, la lógica de la ciencia crítica es olvidada cuando el docente, que es también investigador, asume el rol de enseñar los contenidos, excluyéndola del aula deliberadamente bajo formatos tradicionales. ¿Cuál es la razón?, probablemente algunos prejuicios basados en la suposición errónea de que los estudiantes no tienen la capacidad de desplegar un desarrollo cognitivo similar, por carecer de saberes previos desde donde construir hipótesis y generar la trama conceptual que permite establecer los vínculos epistémicos necesarios para formular y proponer caminos de indagación, por suponer que carecen de habilidades y competencias que lo faciliten o simplemente por asumir que el aula constituye un contexto de aplicación de ese conocimiento que debe ser trasmitido sin cuestionamientos para garantizar que sea aprendido y de igual modo reproducido sin errores.

El problema, entonces, radica en otros prejuicios que desestiman el valor de la praxis reflexiva (Perrenoud, 2004) como herramienta de aprendizaje, de la deducción como didáctica de la enseñanza y del hacer como posibilidad de aprender a partir del error y, en base a éste, reformular experiencias que permitan interpelar reflexivamente a la razón y motivar la formulación de nuevas hipótesis a corroborar desde ese hacer reflexivo que puede ser completado en el contacto y la interacción con el saber de la comunidad.

\section{¿Cómo integrar las prácticas?}

Integrar la investigación, no implica que los estudiantes investiguen, sino trabajar los contenidos en base a problemas que constituyan el objeto de estudio. Esta dinámica les permite plantear hipótesis y proponer metodologías para diagnosticarlos y abordarlos, desarrollando el pensamiento lógico deductivo como parte de la didáctica 
disciplinar. Analizar colectivamente los enfoques emergentes es una estrategia que desarrolla su capacidad de análisis, fomenta el debate, el trabajo en equipo y los sentimientos de pertenencia (Guerra Santana et al, 2019). De esa manera, la trasmisión acrítica del saber es sustituida por la formulación de preguntas que dialogan entre sí, entre quienes las formulan -y responden- y entre éstas y los textos académicos a los que se recurre como soporte, entendiendo que las preguntas no sólo permiten abordar los problemas, sino también revisar las metodologías que permiten verificarlo.

Bajo este formato, los problemas no deberían constituir abstracciones impresas en el papel de un cuaderno, sino situaciones concretas del territorio. En este sentido, son las problemáticas socio-ambientales las más interesantes por la multidimensionalidad de factores que las condicionan y atraviesan (Zavaro, 2020a). La complejidad implícita en lo ambiental (Leff, 2007) lejos de constituir un obstáculo, representa la oportunidad de interdisciplinar la universidad, desarticulando algunas de las jaulas conceptuales (Lander, 2000) que la han fragmentado.

\section{los problemas no deberían constituir abstracciones impre- sas en el papel de un cuaderno, sino situaciones concretas del territorio.}

Enseñar en el territorio impone integrar la extensión -y sus tradiciones vinculares con la comunidad- lo que además de contribuir a su curricularización (Gezmet, 2018), saldando debates interminables, termina por transmutar el formato. Al respecto Bonicatto (2019), provocativamente, sostiene que la extensión tiene que diluirse: es ésta la manera en que no sólo se diluye, sino que se convierte en el diluyente -el solvente por excelencia- que funde y erosiona las fronteras del resto de las prácticas, integrándolas.

El contacto con el territorio transforma al aprendizaje en un aprendizaje situado (Zavaro, 2020b) y contextualizado que supone una enorme riqueza pedagógica porque no sólo focaliza el aprendizaje en las experiencias, expectativas y demandas existentes en torno a las problemáticas que atraviesan a la comunidad, sino que a su vez permite el diálogo con voces en las que vibran otros saberes y experiencias. Aprender en -y con- la comunidad es "per se" transformador porque en ese vínculo se genera un compromiso que no es sólo con el problema ni con el saber construido en ese proceso, sino también con la multiplicidad de conocimientos que interactúan en una suerte de ecología de saberes (de Sousa Santos, 2007), en la que éstos se funden y, fundamentalmente, con ese otro con quien se dialoga y con quienes se establecen lazos que involucran vivencias intensas (Lischetti et al, 2019). 


\section{Aprender en -y con- la comunidad es "per se" transformador por- que en ese vínculo se genera un compromiso que no es sólo con el problema ni con el saber construido en ese proceso, sino tam- bién con la multiplicidad de conocimientos que interactúan en una suerte de ecología de saberes (de Sousa Santos, 2007), en la que éstos se funden $y$, fundamentalmente, con ese otro con quien se dialoga y con quienes se establecen lazos que involu- cran vivencias intensas}

Este tipo de experiencias no sólo le permite a los estudiantes integrarse a esa comunidad, sino también integrar la comunidad académica a una práctica que termina constituyéndose en un puente que integra la universidad al territorio. Al margen de la retórica intencional, son esas características las que convierten a las prácticas integrales en un formato de enseñanza analítico, riguroso, crítico, dialógico, profundamente solidario, y por tanto, pedagógicamente revolucionario.

\section{¿Y entonces, habrá que curricularizar la práctica!}

Si las prácticas integrales son entendidas como espacio de formación, y por lo tanto constituyen prácticas pedagógicas, entonces lo deseable sería injertarlas en la arquitectura de lo que constituye la hoja de ruta del perfil de los futuros egresados: el plan de estudio. Si bien existen experiencias hacia el interior de las cátedras que incluso perduran en la letra de algunos programas de estudio o de ciertos proyectos de extensión, donde se combinan modos de articular estas modalidades y formas integradas de comprender y sintetizar conocimientos, por su relevancia, estos espacios deberían integrar las trayectorias delineadas en la currícula para la obtención del título universitario.

Curricularizar supone la inclusión de un formato particular de enseñanza en el plan de estudios. Siendo que, tal como sugiere Gimeno Sacristán (1995), toda práctica pedagógica gravita en torno al currículum, este tipo de experiencias deberían ser incorporadas a esa estructura. Si las prácticas integrales permiten la integración de funciones, de los saberes que esas funciones aportan y de las metodologías que históricamente las han caracterizado, en tanto fomentan el diálogo entre actores con saberes disímiles, el formato podría constituir un espacio de trabajo que, sin hegemonizar la currícula, configure al menos un bloque que la atraviese a lo largo de la carrera.

El espacio no sólo promete un aprender a partir del hacer y del hacer interpelando los saberes de quienes comparten ese mismo espacio, sino también interpelando los saberes aprendidos en las materias previas o cursadas en paralelo $y$, sobretodo, aquellos aprendidos en diálogo con los sujetos del territorio con quienes dialogan. Siendo que 
las prácticas integrales constituyen una experiencia de aprendizaje donde los problemas se reconfiguran permanentemente como parte de una dinámica absolutamente dialéctica que transforma a quienes participan del formato y al contexto -que es también el territorio-, el tránsito por un espacio de estas características nos acerca, entonces, a una última pregunta que interpela el propósito de consolidar y visibilizar su relevancia como espacio de formación.

El espacio no sólo promete un aprender a partir del hacer y del hacer interpelando los saberes de quienes comparten ese mismo espacio, sino también interpelando los saberes aprendidos en las materias previas o cursadas en paralelo $y$, sobretodo, aquellos aprendidos en diálogo con los sujetos del territorio con quienes dialogan

\section{¿Para qué las prácticas integrales?}

La respuesta a esta pregunta es obvia y ha impregnado sutilmente estas líneas. En la reflexión sobre cómo y por qué curricularizar un formato capaz de integrar a los sujetos que habitan la universidad y transitan, desde ella, los barrios en los que se inserta, subyace el para qué. Como reflexión final, considero que es ahí donde radica el sentido de la práctica, de la necesidad de su curricularización y del sentido de la formación universitaria. La educación superior forma sujetos en los que han de recaer las decisiones futuras de la sociedad y el futuro de la sociedad misma. En ese sentido, es central -como horizonte de futuro-formar sujetos que sean capaces de comprometerse con el conocimiento y la sociedad que integran, formándose integralmente con el convencimiento de ser reconocidos, por esa sociedad, como sujetos integrales. 


\section{BIBLIOGRAFIA}

Andrade, E. y Chacón, E. (2018). Implicaciones teóricas y procedimentales de la clase invertida. PULSO. Revista de Educación, 41, 251-267. Disponible en: https://revistas.cardenalcisneros.es/index.php/PULSO/article/view/306

Bonicatto, M. (2019). Doble Vía. Aportes de la Planificación Estratégica Situacional a la extensión estatutaria de la UNLP. Tesis Doctoral. Inédito. Disponible en: http://sedici.unlp.edu.ar/handle/10915/79848

Bourdieu, P. (1984). Homo academicus. París: Ed de Minuit.

Brunner, J. J. y Flisfish, A. (1989). Los intelectuales y las instituciones de cultura. México: Universidad Autónoma Metropolitana-Azcapotzalco.

Chalmers, A. (2000). ¿Qué es esa cosa llamada ciencia? Madrid: Siglo XXI 3ra Ed.

Chevallard, I. (1999). La transposición didáctica. del saber sabio al saber enseñado. Madrid: Aique.

de Sousa Santos, B. (2007). La universidad en el siglo XXI. Para una reforma democrática y emancipadora de la universidad, Umbrales: Revista del Postgrado en Ciencias del Desarrollo, 15, 13-70.

Gezmet, S. (2018). Curricularización de la extensión universitaria. E+ E: Estudios de Extensión en Humanidades, 5(5) 17-27. Disponible en: https://revistas. psi.unc.edu.ar/index.php/EEH/article/view/19782

Gimeno Sacristán, J. (1995). El currículum: una reflexión sobre la práctica. Madrid: Morata.

Guerra Santana, M., Rodríguez Pulido, J., y Artiles Rodríguez, J. (2019). Aprendizaje colaborativo: experiencia innovadora en el alumnado universitario. Revista de estudios y experiencias en educación, 18(36), 269-281. Disponible en: http://dx.doi.org/10.21703/rexe.20191836guerra5

Kaplún, G. (2014). La integralidad como movimiento instituyente en la universidad. InterCambios. Dilemas y transiciones de la Educación Superior, 1(1), 4451. Disponible en: https://hdl.handle.net/20.500.12008/17061

Lander, E. (2000). ¿Conocimiento para qué?, ¿conocimiento para quién?. Reflexiones sobre la geopolítica de los saberes hegemónicos. Revista Venezolana de Economía y Ciencias Sociales, 6(2), 53-72. Disponible en: http://dx.doi. org/10.22201/cela.24484946e.1999.12-13.52369

Leff, E. (2007). La Complejidad Ambiental, Polis 16, 1-10.

Lischetti, M., Paoletta H. y Sander, J. (2019). El proceso instituyente de las prác- 
ticas socioeducativas territorializadas. Redes de Extensión 5, 51-66. Disponible en: http://revistascientificas.filo.uba.ar/index.php/redes/article/view/6158

López, M. L. (2011). La demanda en extensión universitaria como problema metodológico. Investigación y Ciencia, 52, 63-69. Disponible en: https://www. redalyc.org/pdf/674/67419879008.pdf

Millán, J. D., Polanco, F., Ossa, J. C., Béria, J. S., y Cudina, J. N. (2017). La cienciometría, su método y su filosofía: Reflexiones epistémicas de sus alcances en el siglo XXI. Revista Guillermo de Ockham, 15(2), 17-27. Disponible en: https:// doi.org/10.21500/22563202.3492

Perrenoud, P. (2004). Desarrollar la práctica reflexiva en el oficio de enseñar. Profesionalización y razón pedagógica. Barcelona: Editorial Graó.

Porlán, R. (2002). Constructivismo y Escuela: Hacia un modelo de enseñanza-aprendizaje basado en la investigación. Serie Fundamentos № 4, España: Díada Editoral S.L.

Sandoval, E. A., Aguado, E. y Rogel, R. (2004). Legitimación del conocimiento: el caso de las revistas en ciencias sociales. En: Castillo Pérez (coord.) Desarrollo Científico, Tecnológico y Educación Superior en América Latina. Colección Educación y Sociedad. Zacatecas: Siglo XXI.

Tommasino, H. y Rodríguez, N. (2010). Tres tesis básicas sobre extensión y prácticas integrales en la Universidad de la República, Cuadernos de Extensión. Integralidad: tensiones y perspectivas, 1, 119-142.

Zavaro, C. (2019). Las prácticas de extensión como experiencias de curricularización: entre el debate y la práctica objetivada. Redes de Extensión. 5, 7-22. Disponible en: http://revistascientificas.filo.uba.ar/index.php/redes/article/view/6159

Zavaro, C. (2020a). Saberes ambientales y extensión como sustrato de las prácticas integrales. Revista EXT 12(2), 1-15. Disponible en: https://revistas.unc. edu.ar/index.php/ext/article/view/30566

Zavaro, C. (2020b). Extensión, prácticas integrales y transformación social: la Investigación Acción Participativa (IAP) como fundamento y praxis. Revista Masquedós 5(5), 1-9. Disponible en: http://ojs.extension.unicen.edu.ar/index. php/masquedos/article/view/69 